\title{
An Simplified Reference Frame Selection Configuration Method
}

\author{
Ping Kuang ${ }^{\dagger 1}$ and Jianhua Deng ${ }^{\dagger 2 *}$ \\ School of Information and Software, University of Electronic Science and \\ Technology of China, Chengdu, Sichuan, China, 610054 \\ \{kuangping${ }^{1}$, jianhua.deng $\left.{ }^{2}\right\} @ u e s t c . e d u . c n$
}

\begin{abstract}
Reference Frame Selection (RFS), as reference frame management mechanism in High Efficiency Video Coding (HEVC), plays an important role in constantly developing video coding standard. As RFS has been improved to adapt the introduction of generalized B frame in HEVC. In this paper, a simplified coding configuration method is presented to simplified reference frame management process to cut down on encoding time. This method is applied in real time low-delay encoding configuration with specific simplified rules. Simplified method is conducted on the reference list with biggish Qualitization Parameter $(Q P)$ offset and improves coding efficiency with about $10.5 \%$ reduction on encoding time and only $0.5 \%$ negligible raise on BP-bitrate.

Keywords: HEVC, RFS, depth information, real-time configuration
\end{abstract}

\section{Introduction}

HEVC standard is developed by the oint Collaborative team on video coding and ratified as an international video coding standard to meet the increasing demands for high resolution video.

HEVC is on the block-based hybrid coding framework [1]. Compared with the previous standards, numbers of coding techniques were proposed and accepted to better compress high-difinition video contents. HEVC introduces generalized B frame with more complex GOP structure and reference frame management to reference frame selection (RFS) mechanism [2]. In video coding, a frame encoded by intra prediction is called I frame. And a frame encoded by inter prediction is called P or B frame. For degree of compression, B frame compresses the most, $\mathrm{P}$ frame takes the second place and I frame compresses the least So I frame guarantees the precision of prediction and avoids diffusion of prediction errors, and $\mathrm{P}$ frame and $\mathrm{B}$ frame is used to meet the demands of real-time coding application. Coding configuration file defines the number of I, P and B frame in a basic coding structure called Group of Pictures (GOP) [3]. According to difference of GOP and other coding parameters, for HEVC reference software HM, encoder defines three kind of configuration: intra, low-delay for real-time and randomacces for non-real time application [4]. Hybrid coding structure is applied in low-delay and random-access configuration. In this paper, we mainly focus on simplified method on reference frame management mechanism.

Reference frame management mechanism of video coding goes through lots of improvements. Encoder generally selects the most adjacent restriction frame to be reference frame of current coding frame. However, it is not fit for all the coding structure and application situation, especially application of multi reference frame makes for a huge increase of coding efficiency [5]. The fact that periodically inserting high-quality longterm reference frame contributes to promoting coding performance is proposed in [6], multi- reference frame is proved to be necessary to improve prediction accuracy.

${ }^{1} \uparrow$ Author Contributions: These authors contributed equally. *Corresponding author 
Reference frame selection is modeled as a rate-distortion optimization problem in [7], and then based on reasonable assumption and simplification described in this model, a general situation to this kind of problem is deduced, which is seen as a general method of reference frame setting. " $1+x$ " method for low-delay coding configuration is proposed in [8], "1" stands for a most adjacent reference frame, " $\mathrm{x}$ " stands for $\mathrm{x}$ frames of highquality reference frame, this method saves about $3.6 \%$ bitrate and accepted by HEVC reference software HM.

In this paper, a simplified reference frame configuration method is proposed to cut down on encoding time in real-time configuration. Configuration file defines encoding structure of the whole coding sequence, QP offset that configuration file assign to each frame reflects its prediction precision when encoder works on this frame to some extent [9]. Our experiment on all the reference frames and cut down on some reference frame selection rules of original HEVC with significant reduction on encoding time and reliable bitrate increase. Then depth information of all the reference frames is used in our judgement to determine whether there is one more frame to be removed in reference frame list. It is proved that reference frame with higher QP offset is more probable to be removed.

The rest of the paper is organized as follows: an overview of RFS mechanism and depth definition in HEVC is presented in Section 2. The proposed simplified reference frame selection method and its depth judgement process are given in Section 3. Experiments are provided in Section 4 to verify the proposed method. Finally, we draw the concluding remarks in Section 5.

\section{The Related Work of RFS}

Modern mainstream video coding fiamly uses multiple reference frame technique in reference frame management which definitely infuences coding efficiency [10]. Encoder traverses the entire reference f ane in motion estimation. It improves the precision of prediction. On the other hand, calculations of encoding significantly increase.

This paper aims to find out a simplifed method for real-time encoding configuration which is named "low-delay" among HEVe configuration files. In this configuration, only the first frame in a sequence of frames is encoded by intra configuration as an "I" frame, and its subsequent frames are ail encoded as P frame and generalized B frame. To take full advantage of the high efficiency of "bi-directional" prediction, HEVC introduces generalized B frame, its defines two reference frame lists which store the same reference frames in low-delay configuration and differs a lot from each other in random-access configuration.

HEVC uses Reference Picture Set (RPS) technique to manage the frames in Decoded Picture Buffer (DPB). The reference frames of RPS are classified into two groups: shortterm referenice frame set, and long-term reference frame set [11]. Short-term reference frame set-can be classified into five subsets. Reference of these subsets is classified into three parts RefPicSetStCurrBefore, RefPicSetStCurrAfter and RefPicSetStFoll. Picture Order Count (POC) is defined to denote display order of frames in video coding. RefPicSetStCurrBefore contains the reference frames whose POC is before current coding frame, and RefPicSetStCurrafter contains the reference frames whose POC is after current coding frame. RefPicSetStFoll contains the frames that are not used by current frame. And long-term reference frame set is configured in slice header and not divided into subsets.

Two reference list "list0" and "list1" are created to be used in RFS through two processes: initialize reference lists and modification [12]. The first reference list list0 selects reference frames from subset of short-term reference frame set RefPicSetStCurrBefore, RefPicSetStCurrAfter and RefPicSetLtCurr in order, until the number of reference frames selected into list 0 reaches configured limitation. And the 
second reference list list1 selects reference frames from RefPicSetStCurrAfter, RefPicSetStCurrBefore and RefPicSetLtCurr in order. If the number of frames in subset RefPicSetStCurrAfter is set to 0, list0 and list1 are the same, as generalized B frame defines. Modification for reference list is not essential and flags exit in bit stream to indicate whether modification should be performed or not. Modification process can adjust the position of reference frames as expected.

\section{Proposed Simplified Reference Frame Selection Method}

To reduce computational complexity of encoder, statistics of reference frames distribution of pictures in GOP are made in the " $1+3$ " coding structure of low-delay configuration. The flowchart of proposed simplified RFS method is given in Figure1.

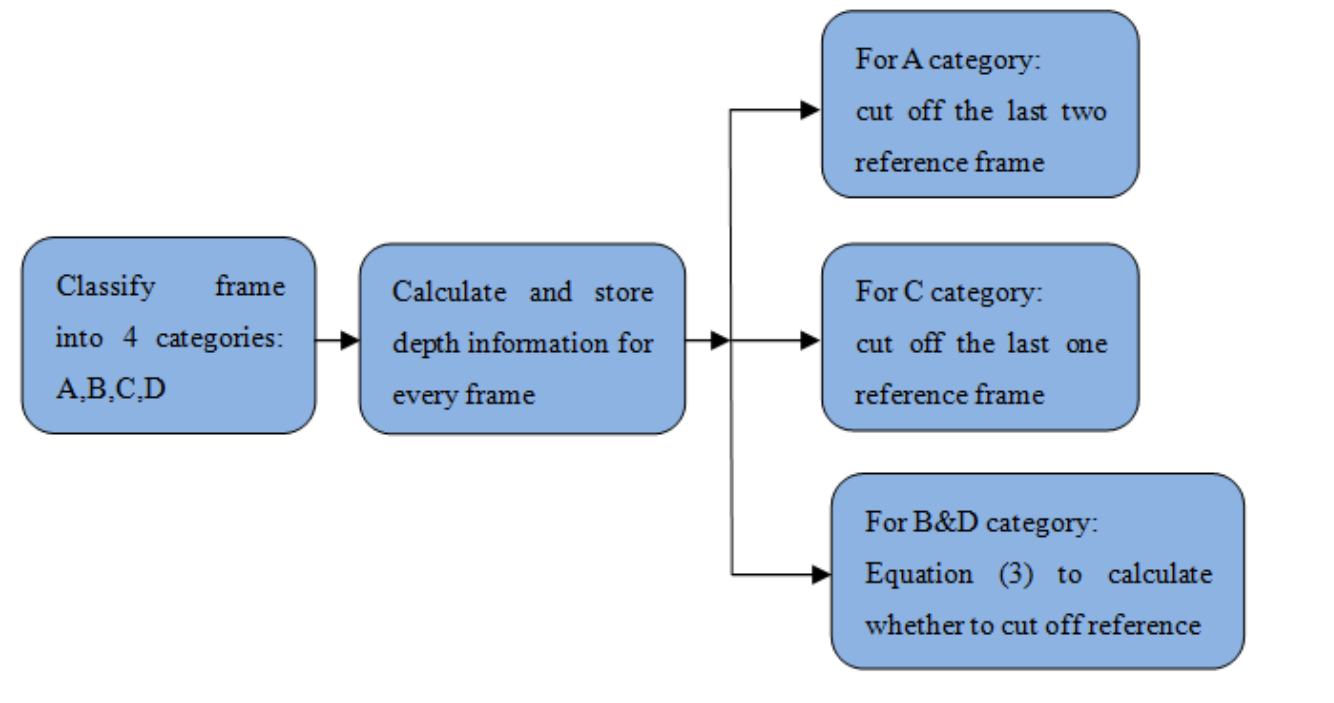

Figure 1. The Fowchart of Proposed Simplified RFS Method

\subsection{Simplified Low-Delay Configuration Method}

Low-delay confíguration nncludes two kind of encoding structure: IBBB and IPPP, for generalized B frame an $\mathrm{P}$ trame, respectively. IBBB encoding structure can be described in Table. 1. POC of reference picture in Table. 1 indicates the POC of reference frame of current encoding frame. The position of reference frames and GOP structure correlations of encoding frame and reference frame is shown in Figure2. The first frame whose POC is 0 is processed by intra prediction as an "I" frame. Every bar's height corresponding to every frame indicates the value of QP offset when encoding this frame [13]. QP offset can be $1,2,3,4$ corresponding to different height. 


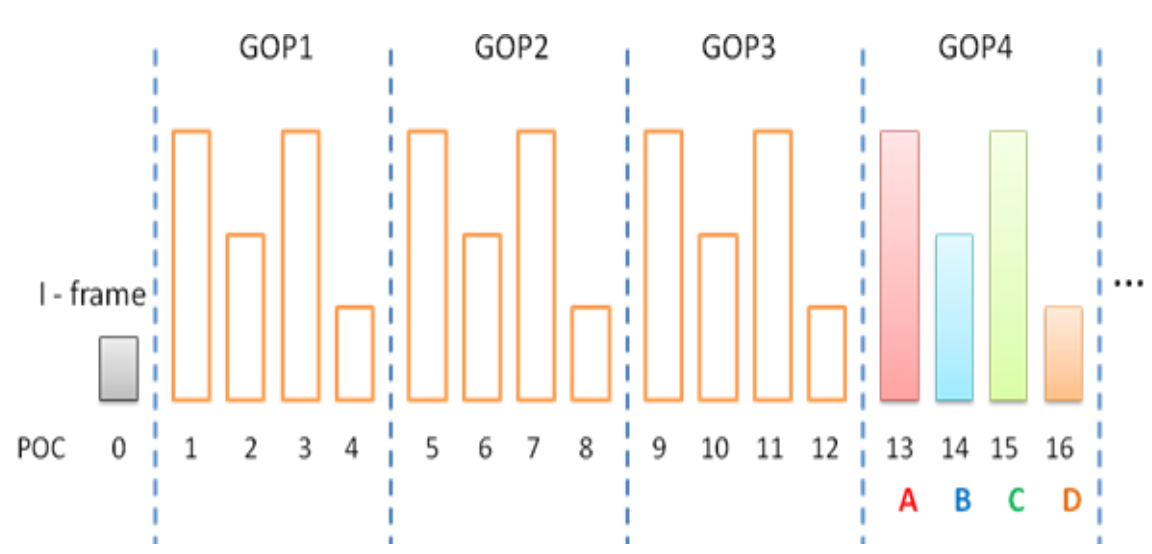

Figure 2. GOP Structure of Low-Delay Configuration in HEVC

Table 1. Low-Delay Configuration IBBB Structure Reference Frame Setting

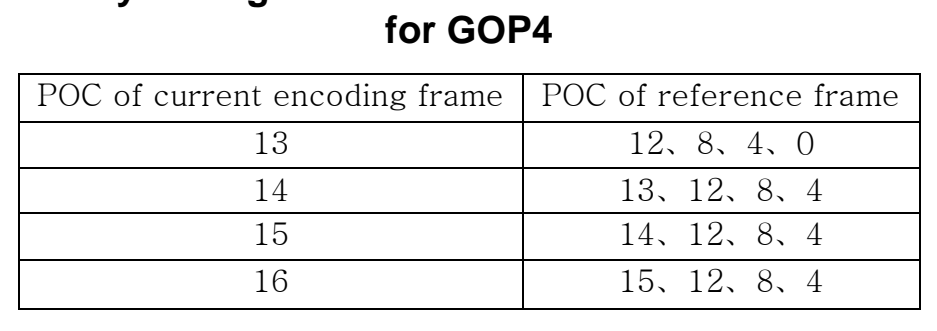

The four frames in GOP4 select theicreference frames ltilizing a fixed " $1+3$ " structure, meaning one frame which is most adjacent to curtent frame and three high-quality frames whose QP offset is the least in therrespective_GOP, usually located in the end of forward GOP as general low-delay configuration dêfines [14].

Four pictures of a GOP can be classified into four parts denoted by A, B, C, and D. Four reference frames of every kind of picture are denoted by R0, R1, R2, R3, respectively. The percentage of curtent frame encoded by intra prediction is denoted by Pintra. The percentage of current frame selecting reference frame labelled $R i$ is denoted by Pi. It is obviously that,

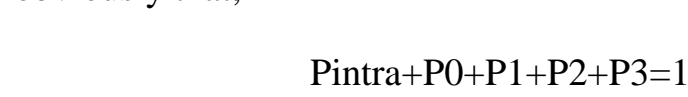

Statistics experiments are performed in HEVC reference software HM, version 13, low-delay configuration with QP equal to 27. First 113 frames of 10 Test sequences are selected as experimental samples. Reference frames in GOP start to possess universality from the fourth GOP, with POC starting from 12.

Statistics of reference frames distribution are shown in Table.2. Average percentage distribution can be described in Figure3.

Table 2. Percentage Distribution of Reference Frame Combinations for Pictures of Class A

\begin{tabular}{|c|c|c|c|c|c|}
\hline combination & $\begin{array}{c}\text { Pintra+ P0 } \\
(\%)\end{array}$ & $\begin{array}{c}\text { Pintra+ P0+ P1 } \\
(\%)\end{array}$ & $\begin{array}{c}\text { Pintra+ P0+ P2 } \\
(\%)\end{array}$ & $\begin{array}{c}\text { Pintra+ P0+ P3 } \\
(\%)\end{array}$ & $\begin{array}{c}\text { Pintra+ P0 } \\
+ \text { P1+ P2 } \\
(\%)\end{array}$ \\
\hline BasketballDrive & 97.26 & 99.02 & 98.34 & 97.47 & 99.35 \\
\hline RaceHorses & 93.41 & 97.15 & 94.28 & 94.51 & 97.20 \\
\hline BasketballDrill & 90.29 & 95.26 & 92.08 & 93.49 & 97.34 \\
\hline BasketballPass & 97.33 & 98.55 & 97.00 & 96.07 & 98.79 \\
\hline BlowingBubbles & 96.86 & 98.19 & 97.51 & 96.41 & 98.90 \\
\hline
\end{tabular}




\begin{tabular}{|c|c|c|c|c|c|}
\hline BQSquare & 91.30 & 97.67 & 94.26 & 92.62 & 97.71 \\
\hline FourPeople & 91.43 & 95.90 & 98.04 & 96.35 & 96.12 \\
\hline Johnny & 91.67 & 94.45 & 93.22 & 93.57 & 94.63 \\
\hline SlideShow & 95.93 & 97.21 & 96.04 & 97.05 & 98.33 \\
\hline Traffic & 91.02 & 97.98 & 92.75 & 94.29 & 98.49 \\
\hline Average & 93.65 & 97.14 & 95.35 & 95.18 & 97.69 \\
\hline
\end{tabular}

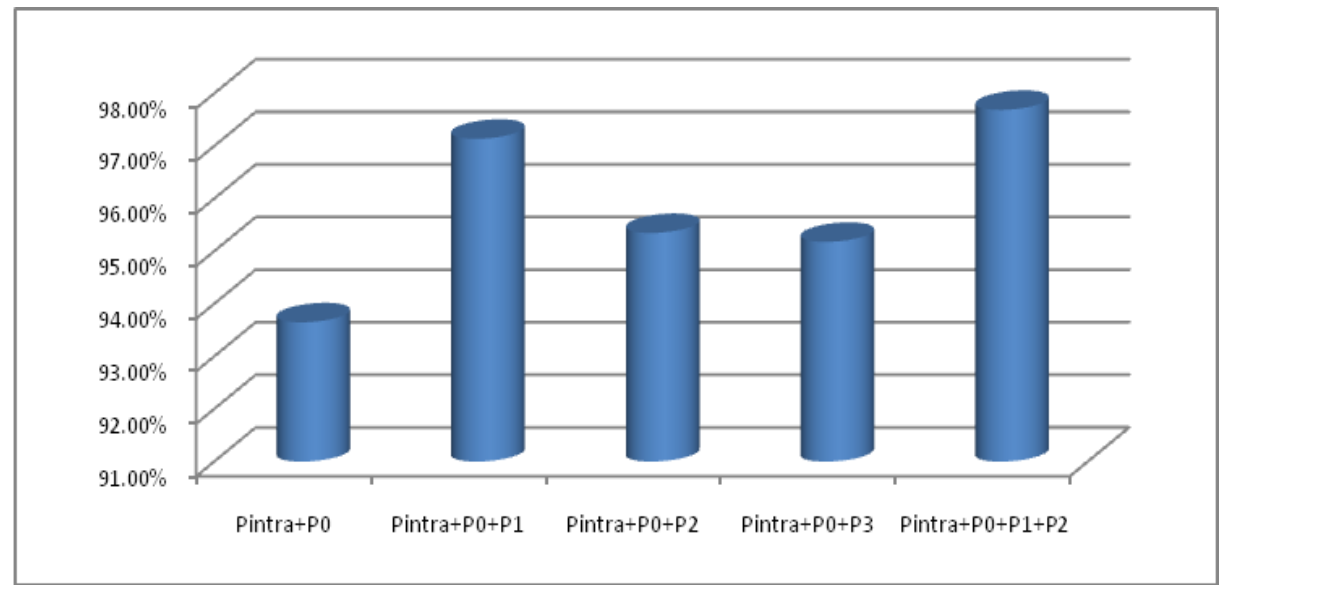

Figure 3. Average Percentage Distribution of Reference Frame Combinations for Pictures of Class A

As is shown in Table 3 and Figure 3 , when referene frames are set as R0+R1, seven sequences in ten test sequences indicate more than $97 \%$ percentage hit rate of reference frame selection, and $\mathrm{R} 0+\mathrm{R} 1+\mathrm{R} 2$ combination does not significantly increase hit rate of selection with the percentage raise about $0.6 \%$ So it is reasonable to select $R 0+R 1$ or $\mathrm{R} 0+\mathrm{R} 1+\mathrm{R} 2$ as simplified referenee frame selection settings for pictures of class $\mathrm{A}$. In our proposed method, R0+R1 are selected considering real-time encoding demand for HEVC.

Furthermore, statistic experiment are also performed on pictures of class B, C, D, and the results indicate that class $\mathrm{C}$ presents similar regulations with class $\mathrm{A}$, as they both correspond to the Diggest QP offse in GOPs, which means a relatively cursory prediction compared with frames with smaller QP offset. Experimental statistics for pictures of class $\mathrm{C}$ are given in Table 3 and Figure 4.

Table 3. Percehtage Distribution of Reference Frame Combinations for Pictures of Class C

\begin{tabular}{|c|c|c|c|c|c|}
\hline combination & $\begin{array}{l}\mathrm{Pa}+\mathrm{P} 0+\mathrm{P} 1 \\
(\%)\end{array}$ & $\begin{array}{c}\text { Pintra+ P0+ P2 } \\
(\%)\end{array}$ & $\begin{array}{c}\text { Pintra+ P0+ P3 } \\
(\%)\end{array}$ & $\begin{array}{c}\text { Pintra+ } \mathrm{P} 0+\mathrm{P} 1+\mathrm{P} 2 \\
(\%)\end{array}$ & $\begin{array}{c}\text { Pintra+ P0 } \\
+\mathrm{P} 1+\mathrm{P} 3 \\
(\%)\end{array}$ \\
\hline BasketballDrive & 93.77 & 83.90 & 82.56 & 97.45 & 96.46 \\
\hline RaceHorses & 94.61 & 91.83 & 88.30 & 98.39 & 94.19 \\
\hline BasketballDrill & 90.29 & 91.06 & 88.95 & 91.29 & 95.38 \\
\hline BasketballPass & 95.33 & 86.62 & 85.81 & 98.05 & 97.37 \\
\hline BlowingBubbles & 94.36 & 87.24 & 87.35 & 99.48 & 95.74 \\
\hline BQSquare & 82.68 & 77.36 & 73.94 & 94.19 & 88.09 \\
\hline FourPeople & 95.83 & 88.40 & 87.64 & 99.03 & 98.54 \\
\hline Johnny & 92.19 & 84.45 & 85.64 & 98.80 & 95.02 \\
\hline SlideShow & 93.37 & 82.57 & 82.76 & 97.26 & 95.99 \\
\hline Traffic & 94.27 & 90.57 & 83.76 & 97.11 & 92.48 \\
\hline Average & 92.67 & 86.40 & 84.47 & 97.10 & 94.93 \\
\hline
\end{tabular}




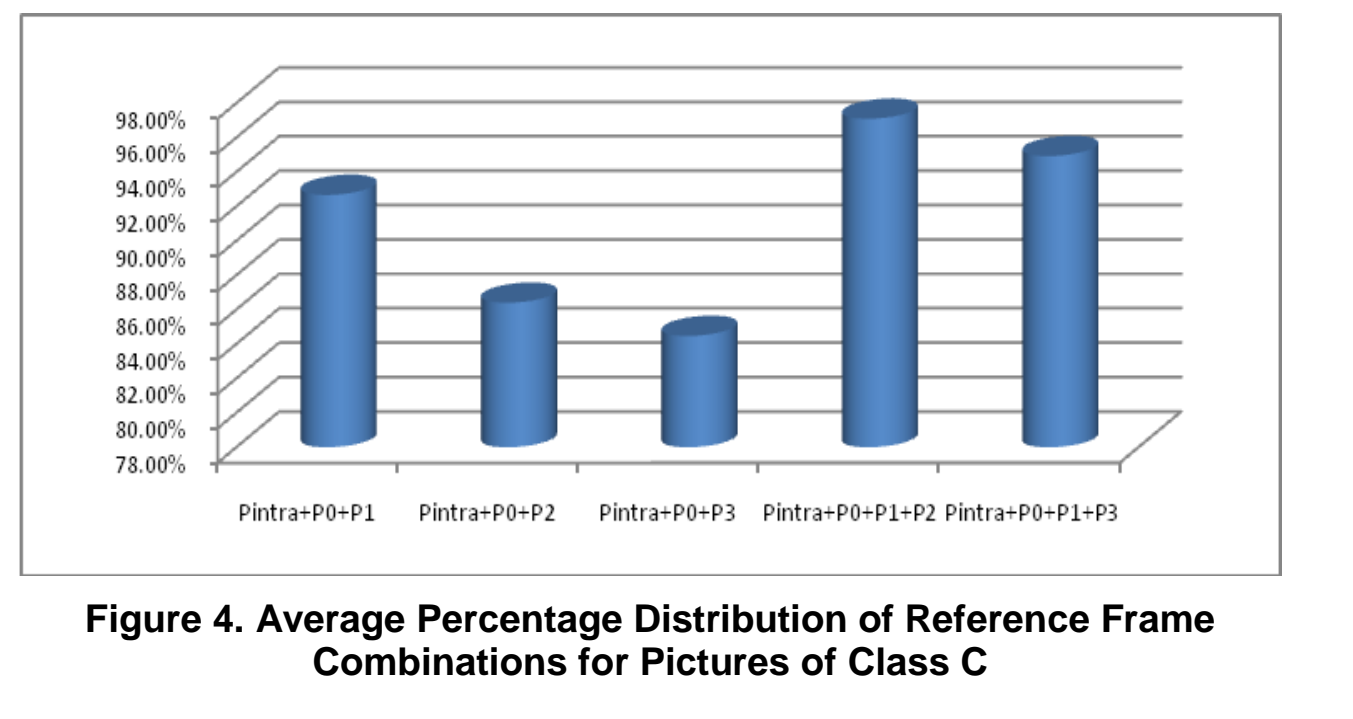

It proves that the combination of $\mathrm{R} 0+\mathrm{R} 1+\mathrm{R} 2$ indicate over $97 \%$ hit rate of reference selection, far more than other groups, and it is obvioas that $R 0+R 1$ is not enough to guarantee the precision of prediction with the bit rate less than $93 \%$. Above all, in proposed simplified method, R0+R1 is retained fo pictures of class $A$ and $R 0+R 1+R 2$ is retained for class C. New configuration of RFS in proposed method can be described in Table. 4.

Table 4. New Configuration of Reference Frame Setting for GOP4 in Proposed Method

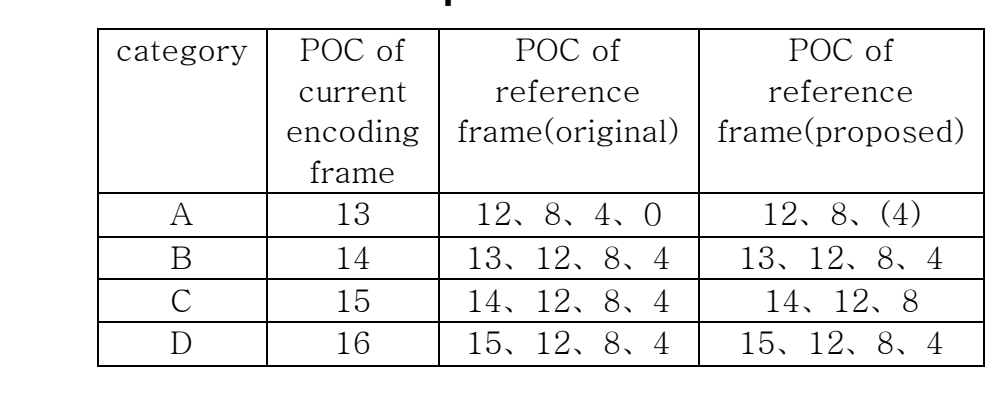

\subsection{Depth Judgment Simplification for High Quality Reference Frame}

Each video frame is divided into a sequence of Coding Tree Unit (CTU) to be processed in splitting process of intra and inter prediction. Each CTU can be recursively divided into four CUs with equal size, thus forming a coding quad-tree splitting structure [15]. The CUs size may range from $8 \times 8$ to $64 \times 64$ luma samples as configuration file defires. 


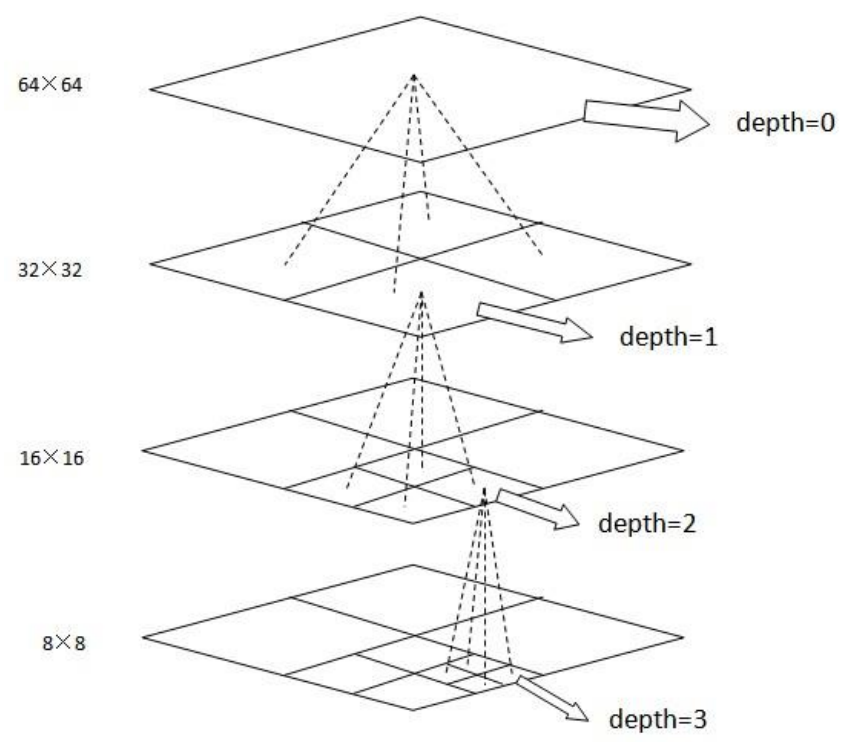

Figure 5. Depth Definition Corresponding tô CU size

$\mathrm{CU}$ is basic region of coding process, HEVC defines a variable depth corresponding to each kind size of CU. Depth is 0 when CU size equals CTU usually is $64 \times 64$. As is shown in Figure5, depth plus one as long as $\mathrm{CU}$ is divided into four equally sized subCUs until sub-CUs' size equals the minimun size $8 \times 8$ conesponding to depth that is equal to 3. Depth of a frame reflects its prediction precision, in coding process, providing basis to determine whether a frame is high quality or not together with QP offset assignment regulations [16].

CU splitting depth reflects the prediction quality along with its correlation with QP. Depth of every frame derives from the average depth of all the $4 \times 4$ pixels blocks in this frame, which can be calculated as

$$
\text { - depth } \left.\frac{\sum_{0}^{M-1} \sum_{0}^{N=} \operatorname{lepth}_{4 \times 4}(i) / N}{N}\right) / M
$$

where depthrame denotes the depth information of current encoding frame, depth $4 \times 4$ (i) denotes a $4 \times 4$ pix lels block inside a CU, $\mathrm{N}$ denotes the number of $4 \times 4$ block in corresponding CTU, M denotes the number of CTUs in corresponding frame.

Simplified setting method for pictures of class B and class D derives from depth relationship of reference frames. Depth of reference frames that are labelled R1, R2, and $\mathrm{R} 3$ are selected to be compared with each other. These frames are assigned with the same QP offset as configured.

For fames of class B and class D, three long term reference frames are included in reference 1ist of low-delay configuration. Depth information is utilized to distinguish the quality of frames in reference list. If one of them is calculated by its depth to be not highquality enough compared to the other two reference frame, this frame is removed from reference list.

Experiments are conducted to determine the threshold used in determining how far current frame which is labelled Ri is away from the average depth of three frames' depth which is denoted by Taway ranging from 0.1 to 0.4 and how close the other two reference frames are close to each other denoted by Tclose ranging from 0.01 to 0.04 . Tclose is scaled by variance of average depth of the other two reference frames. Statistics of the percentage of best reference frame still included in simplified reference list are shown in Table. 5. 


\section{Table 5. Statistic of the Percentage of Best Reference Frame Included in Simplified Reference List}

\begin{tabular}{|c|c|c|}
\hline $\mathrm{T}_{\text {away }}$ & $\mathrm{T}_{\text {close }}$ & Hit rate of simplified reference list (\%) \\
\hline 0.1 & \multirow{4}{*}{0.01} & 97.1 \\
\hline 0.2 & & 96.3 \\
\hline 0.3 & & 74.5 \\
\hline 0.4 & & 61.3 \\
\hline 0.1 & \multirow{4}{*}{0.02} & 95.1 \\
\hline 0.2 & & 97.5 \\
\hline 0.3 & & 84.3 \\
\hline 0.4 & & 77.2 \\
\hline 0.1 & \multirow{4}{*}{0.03} & 92.1 \\
\hline 0.2 & & 89.3 \\
\hline 0.3 & & 75.4 \\
\hline 0.4 & & 53.9 \\
\hline 0.1 & \multirow{4}{*}{0.04} & 79.2 \\
\hline 0.2 & & 66.4 \\
\hline 0.3 & & \\
\hline 0.4 & & 7 \\
\hline
\end{tabular}

When Taway equals 0.2 and Tclose equal 002 , simplified reference list shows the best hit rate of including the original best reference frame $S 0$ specific criterion is made for simplification for pictures of class B and lass D, when their depth correlations meet the rule, specific reference frame labelled $R i$ can be removed from original method. The criterion $C$ is given by

where $c 1$ is given by

$$
\text { depth }_{R i}<\frac{\text { depth }_{R j}+\text { depth }_{R k}+\text { depth }_{R i}}{3}-0.2
$$

And $c 2$ is calculated as

$$
\left(\frac{d e p t h_{R j}^{2}+d e p t h_{R k}^{2}}{2}\right)-\left[\frac{d e p t h_{R j}+d e p t h_{R k}}{2}\right]^{2}<0.02
$$

where depth $i$ denotes the depth of reference frame which is being calculated, $i$ values from 1 to 3 , denoting corresponding frame of current reference list when calculating. $R j$ and $R k$ denotes the other two reference frame in the same reference list besides $R i$, $\operatorname{depth} R j$ and depth $R k$ denote depth of the two reference frame, respectively.

If depth information meets specific criterion given in equation(3), $R i$ is considered as a elatively cursory reference compared with the other two reference frame and immediately removed from original reference frame set, as the number of reference frames of class B and class D pictures is cut down from 4 to 3 , impressively saving encoding time.

\section{Experimental Results}

In this section, the performance of the proposed simplified reference frame selection method is evaluated in terms of the difference in computational time Average Saving Time Percentage (ASTP), Bjontegaard Delta bit rate (BDBR) and BD-PSNR (dB) [17]. The performance gain or loss is measured with respect to the HEVC reference software 
platform (HM 12.0) [18]. The experiments are implemented based on the "encoder_lowdelay_main" (LB-Main) settings [19] as stipulated by the common condition which was proposed in [20]. The quantization parameters values are set to 22, 27, 32 and 37 for a wide range of qualities and bit-rates. The simulation is performed on a computer with an Intel Core i7-2600 $3.4 \mathrm{GHz}$ processor. The time savings are by a customized variable ASTP calculated as

$$
A S T P=\frac{\text { Enc.Time }_{H M 12.0}-\text { Enc.Time }_{\text {Proposed }}}{\text { Enc.Time }_{H M 12.0}} \times 100 \%
$$

where Enc.TimeHM12.0 denotes the average encoding time through all the QP value of original HEVC Reference Software HM12.0, and Enc.TimeProposed denotes encoding time of the proposed simplified reference frame selection method. Table. 6 shows the results of single test sequence base on the "LB-Main" setting with proposed method applied.

Table 6. Proposed Method Experimental Results of Single Sequence in LBMain Configuration

\begin{tabular}{|c|c|c|c|c|}
\hline \multirow{2}{*}{ test sequences } & \multicolumn{2}{|c|}{ Increase of BDBR of every compont $(\%)$} & ASTP \\
\cline { 2 - 5 } & $\mathrm{Y}$ & $\mathrm{U}$ & $\mathrm{V}$ & (\%) \\
\hline BasketballDrive & 0.4 & 0.9 & 0.7 & 9.3 \\
\hline RaceHorses & 0.1 & 0.6 & 0.3 & 12.9 \\
\hline BasketballDrill & 0.9 & 1.3 & 0.8 & 10.7 \\
\hline BasketballPass & 0.2 & 0.4 & 0.5 & 9.2 \\
\hline BlowingBubbles & 0.3 & 0.2 & 0.2 & 9.9 \\
\hline BQSquare & 0.4 & -0.9 & -0.2 & 8.2 \\
\hline FourPeople & 0.3 & 0.8 & 0.0 & 11.0 \\
\hline Johnny & 0.3 & 0.3 & -0.4 & 12.1 \\
\hline SlideShow & 0.2 & 0.1 & 0.1 & 11.8 \\
\hline Traffic & 1.1 & 1.0 & 0.7 & 10.3 \\
\hline Averagte & 0.4 & 0.41 & 0.27 & 10.54 \\
\hline
\end{tabular}

Experiments are also performed on different kinds of sequences with different specific definition. The range of definition from $416 \times 240$ to $2560 \times 1600$, and its average increase of BDBR, average ASTP and total average are shown in Table. 7.

Experimental results indicate that proposed method brings more than $10 \%$ reduction on encoding time. And the BDBR increase of all the color components are limited no more than $0.5 \%$. Proposed method shows great adaptability and stability referring to the effect that experiment performed on all kinds of video sequences with different definition, with impressive redúction on coding time and negligible BDBR increase in average.

\section{Table 7. Proposed Method Experimental Results on Different Definition} Video Sequences in LB-Main Configuration

\begin{tabular}{|c|c|c|c|c|}
\hline \multirow{2}{*}{ variable test } & \multicolumn{3}{|c|}{$\begin{array}{c}\text { Average increase of BDBR of every } \\
\text { component }(\%)\end{array}$} & $\begin{array}{c}\text { ASTP } \\
\text { sequence definition }\end{array}$ \\
\cline { 2 - 4 } & $\mathrm{Y}$ & $\mathrm{U}$ & $\mathrm{V}$ & \\
\hline $416 \times 240$ & 0.64 & 0.59 & 0.73 & 9.5 \\
\hline $832 \times 480$ & 0.60 & 0.82 & 0.34 & 13.2 \\
\hline $1280 \times 720$ & 0.81 & 0.31 & 0.55 & 10.1 \\
\hline $1920 \times 1080$ & 0.23 & 0.17 & 0.09 & 11.3 \\
\hline
\end{tabular}




\begin{tabular}{|c|c|c|c|c|}
\hline $2560 \times 1600$ & 0.77 & 0.82 & 0.72 & 8.5 \\
\hline Total Average & 0.61 & 0.54 & 0.49 & 10.52 \\
\hline
\end{tabular}

\section{Conclusion}

In this paper, a simplified reference frame selection method is proposed. The implicit correlation between the prediction precision of reference frame settings and splitting depth along with QP is investigated. Reliable reference frame is cut off from original RFS method for these frames with the biggest QP offset which indicate relatively cursory prediction. And depth information is calculated and stored for every frame, and is utilized to make judgement for reference frames with smaller QP offset. The experimental results show that the proposed method reduces the encoding time significantly and has a negligible loss of prediction precision at the same time.

\section{Acknowledgement}

This work is supported by the Science and Technology Supporting Program of Sichuan Province, China (No. 2015GZ0358), and Science and Technology Support Program of China (No. 2012BAH44F02), and Smart City Innovation and Opening rund of UESTC (No. RWS-CYHKF-02-20150004).

\section{References}

[1] Ahmad, X. Wei, Y. Sun and Y. Zhang, "Video tanseoding: ân 6reryiew of various techniques and research issues", IEEE Trans. Multimedia, vol. 7, (2005), pp. 793-804.

[2] T. Wiegand, G. Sullivan, G. Bjontegaard and A. Luthra, "Oyerview of the H.264/AVC video coding standard", IEEE Transaction on Circuits and Systems for Viceo Technology, vol. 13, no. 7, (2003), pp. 560-576.

[3] G. J. Sullivan, J. R Ohm, W. J Hahand T. Wiegand, Overview of the high efficiency video coding (HEVC) standard", IEEE Transaction on Cireuit and Systems for Video Technology, vol. 22, no. 12, (2012).

[4] "High efficiency video coding (HEVC/H,265), ITU-T Telecommunication Standardization Sector of ITU, (2013).

[5] F. Bossen, B. Bross, R. Shring and D. Flynn, "HEVC complexity and implementation analysis", IEEE Transaction on Circuits and Systems for Video Technology, vol. 22, no. 12, (2012), pp. 1684-1695.

[6] V. Chellappa, P.C. Cosman and G. M. Voelker, "Dual frame motion compensation with uneven quality assignment, Circuits and Systems for Video Technology", IEEE Transactions on, vol. 18, no. 2, (2008), pp. $249-256$.

[7] H. L, B. Li and J. Xu Rate-distortion optimized reference picture management for high efficiency video coding", IEEE-Transaction on Circuits and Systems for Video Technology, vol. 22, no. 12, (2012), pp. 1844-1857.

[8] B. Li, J. Xu, P.Wu and Q. Li, "Encoding optimization to improve coding efficiency for low delay cases", JCTVC-F701, Torino, IT, (2011).

[9] Z. X Chen and S. S Goto, "A QP and partition-size statistic based fuzzy algorithm for fast inter\&intra mode decision in video coding", The 2th International Congress on Image and Signal Processing, (2009).

[10] L. Bin, "Optimized reference frame selection for video coding by cloud", IEEE the 13th International Workshop on Multimedia Signal Processing (MMSP), (2011).

II) W. Han, J. Min, I. K. Kim, E. Alshina, A. Ashin, T. Lee, J. Chen, V. Seregin, S. Lee, Y. M. Hong, M. S. Cheon, N. shlyakhow, K. McCann, T. Davies and J. H. Park, "Improved video compression efficiency through flexible unit representation and corresponding extension of coding tools", IEEE Trans. Circuits Syst. Video Technology, vol. 20, no. 12, (2010), pp. 1709-1720.

[12] K. Ugur, K. Andersson, A. Fuldseth, G. Bjontegaard, L. P. Endresen, J. Lainema, A. Hallapuro, J. Ridge, D. Rusanovskyy, C. Zhang, A. Norkin, C. Priddle, T. Rusert, J. Samuelsson, R. Sjoberg and Z. Wu, "High performance, low complexity video coding and the emerging HEVC standard", IEEE Trans. Circuits Syst. Video Technology, vol. 20, no. 12, (2010), pp. 1688-1697.

[13] J. R. Ohm, G. J. Sullivan, H. Schwarz, T. K. Tan and T. Wiegand, "Comparison of the coding effciency of video coding standards including high efficiency video coding (HEVC)", IEEE Transaction on Circuits and Systems for Video Technology, vol. 22, no. 12, (2012).

[14] L. C. Soon, "Thet NSM, Reference lists for low delay settings", JCT-VC Document, JCTVC-F433, Torino, Italy, (2011). 
[15] P. Helle, S. Oudin and B. Bross, "Block merging for quadtree-based partitioning in HEVC", IEEE Transactions on Circuits and Systems for Video Technology, (2012).

[16] J. Xiong, "Fast coding unit selection algorithm for high efficiency video coding intra prediction", Optical Engineering, vol. 52, no. 7, (2013), pp. 071 504-071 504.

[17] G. Bjontegaard, "Calculation of average PSNR differences between RD curves", in No. ITU-T SC16/Q6, VCEG-M33, Austin, TX, USA, (2001).

[18] "JCT-VC HEVC reference software version HM 12.0", available online at https://hevc.hhi.fraunhofer.de/svn/Software/tags/HM-12.0.

[19] "JCT-VC, HEVC reference software manual", JCTVC-J0470, JCT-VC Meeting, Stockholm, Sweden, (2012).

[20] F. Bossen, "Common HM test conditions and software reference configurations", document JCTVCL1100, ITU-T/ISO/IEC Joint Collaborative Team on Video Coding (JCT-VC), (2013).

\section{Authors}

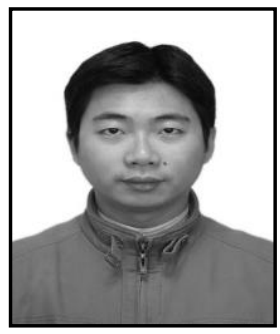

Ping Kuang, Associate professor in University of Electronic Science and Technology of China(UESTC). In 2000, 2003 and 2006, he obtained his Bachelor, Master and Ph.D. in UESTC respectively. His interests include image processing and cloud computing.

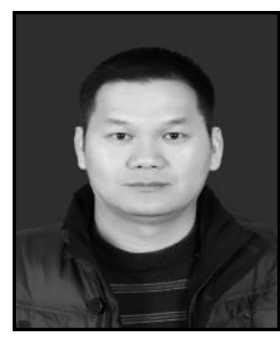

Jianhua Deng, he graduated in information security from the University of Electronic Science and Technology of China (UESTC), China, in 2006. After graduated, he joined the School of Computer Science and Engineerng at UESTC as a staff. From 2009 to 2014, he was a Ph.D. Student in Dúblin Institute of Technology (DIT) in Ireland and received Ph. . degree in electrical engineering from DIT in 2014. Now, he is a leeturer in the School of Information and Software Engineering at UESTC. His research activities are focused on wireless communieation and video streaming.

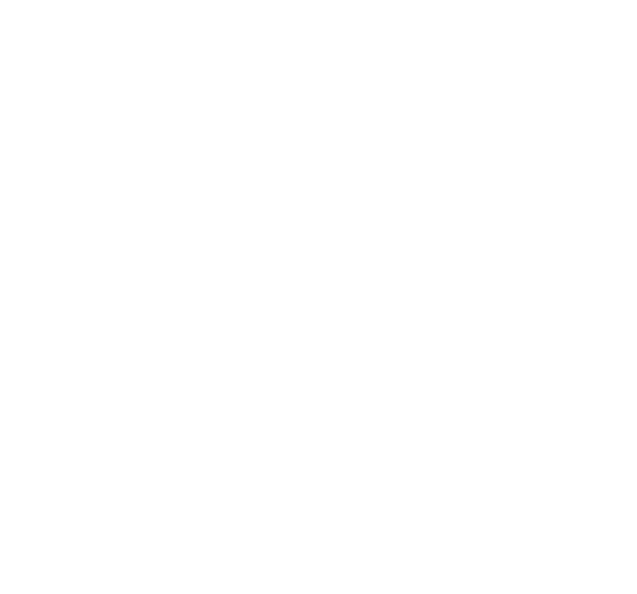


International Journal of Multimedia and Ubiquitous Engineering

Vol.11, No.9 (2016)

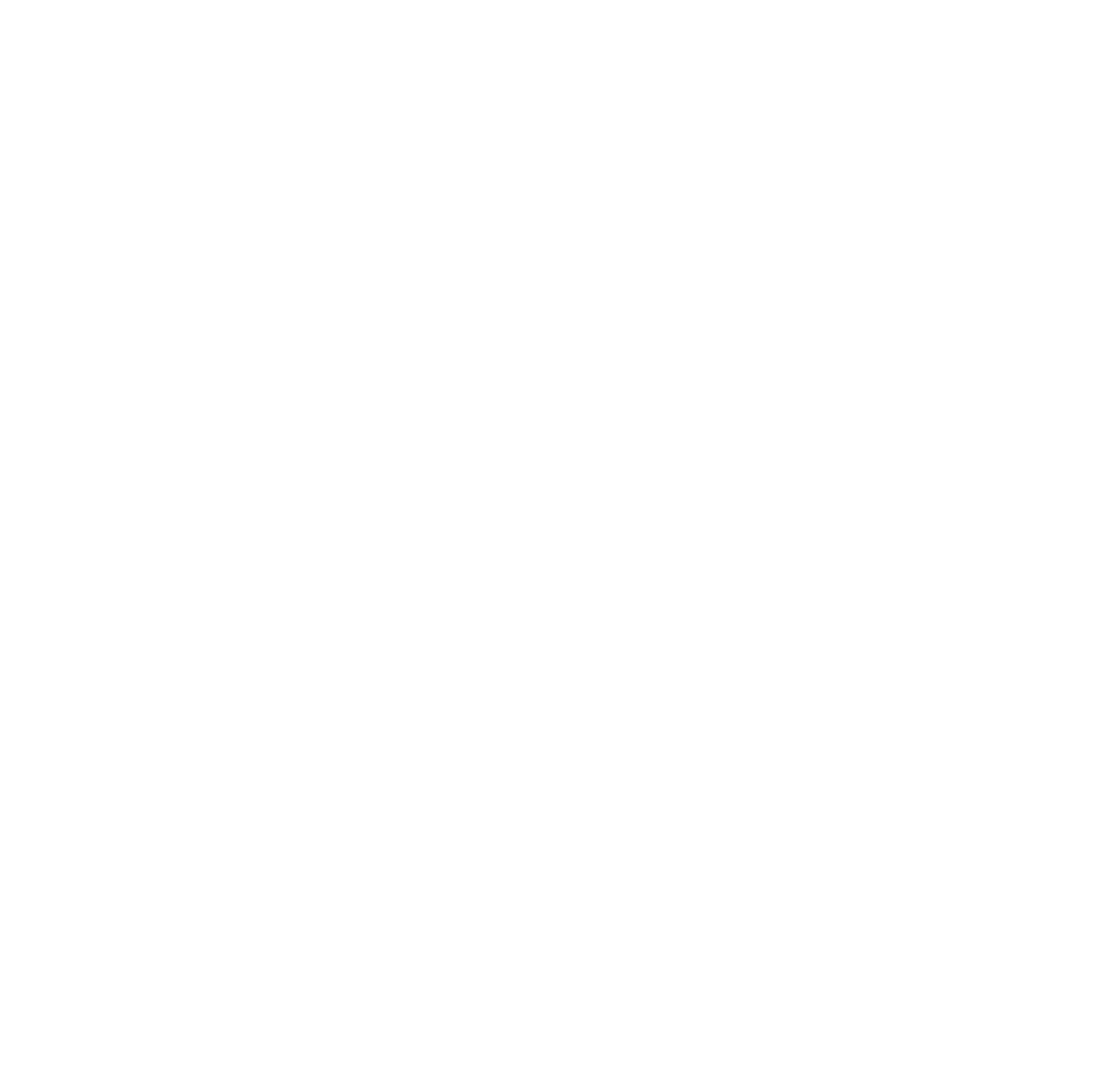

